\title{
Well-Posedness for Generalized Set Equilibrium Problems
}

\author{
Yen-Cherng Lin \\ Department of Occupational Safety and Health, College of Public Health, China Medical University, Taichung 40421, Taiwan
}

Correspondence should be addressed to Yen-Cherng Lin; yclin@mail.cmu.edu.tw

Received 14 July 2013; Accepted 13 September 2013

Academic Editor: Jen-Chih Yao

Copyright (C) 2013 Yen-Cherng Lin. This is an open access article distributed under the Creative Commons Attribution License, which permits unrestricted use, distribution, and reproduction in any medium, provided the original work is properly cited.

We study the well-posedness for generalized set equilibrium problems (GSEP) and propose two types of the well-posed concepts for these problems in topological vector space settings. These kinds of well-posedness arise from some well-posedness in the vector settings. We also study the relationship between these well-posedness concepts and present several criteria for the well-posedness of GSEP. Our results are new or include as special cases recent existing results.

\section{Introduction and Preliminaries}

Let $X, Y, Z$ be three topological vector spaces, $K$ a nonempty closed convex subset of $X, C \subset Y$ a closed convex and pointed cone with apex at the origin, and int $C \neq \emptyset$; that is, $C$ is properly closed with nonempty interior and satisfies $\lambda C \subseteq C$, for all $\lambda>0 ; C+C \subseteq C$; and $C \cap(-C)=\{0\}$.

The set-valued mapping $f: Z \times K \times K \rightrightarrows Y$ satisfies $f(s, x, x)=\{0\}$ for all $x \in K$ and for all $s \in Z$, and a setvalued mapping $T: K \rightrightarrows Z$ is given. The generalized set equilibrium problem (GSEP) is to find an $\bar{x} \in K$ with some $\bar{s} \in T(\bar{x})$ such that

$$
f(\bar{s}, \bar{x}, y) \cap(-\operatorname{int} C)=\emptyset \quad \forall y \in K .
$$

We denote the set of all solutions for (GSEP) by $\Omega$.

The concept of well-posedness is inspired by numerical methods producing optimizing sequences for optimization problems [1]. There are many cases so that the solutions may not be unique for a minimization problem. A naturally generalized concept of well-posedness which permits the existence but not uniqueness of minimizers and the convergence of some subsequence of every minimizing sequence toward a minimizer. Other more general notions of well-posedness have been introduced in [2] and there are many others in the literature; see, for example, [3-15]. Our main purpose is to derive some properties of well-posedness for the generalized set equilibrium problems. We also study the relations between these properties.
A minimizing mapping $\Phi: Z \times X \rightrightarrows Y$ is defined by

$$
\Phi(s, x)=\operatorname{Min}_{w} f(s, x, K)
$$

for all $(s, x) \in Z \times X$, where $\operatorname{Min}_{w} A=\{\eta \in A: A \cap(\eta$-int $C)=$ $\emptyset\}$ and $f(s, x, K)=\bigcup_{y \in K}\{f(s, x, y)\}$ for all $(s, x) \in Z \times X$. Assume that $\operatorname{Dom}(\Phi) \neq \emptyset$. We note that $0 \in f(s, x, K)$ for all $(s, x) \in Z \times X$ since $f(s, x, x)=\{0\}$ for all $x \in K$ and for all $s \in$ $Z$. $\mathfrak{N}_{X}\left(x_{0}\right)$ denotes the collection of neighborhoods around $x_{0}$ in $X$, similar notations for $\mathfrak{N}_{Y}\left(y_{0}\right)$ and $\mathfrak{N}_{Z}\left(s_{0}\right)$. For any mapping $F, F(A)$ denotes the union $\bigcup_{x \in A} F(x)$.

We propose some properties that can be easily derived from the definition. For the sake of clarity, we give the following proof.

Lemma 1. (i) $\Phi(s, x) \cap \operatorname{int} C=\emptyset$ for all $x \in K$ and for all $s \in T(x)$.

(ii) $\bar{x} \in \Omega$ with $\bar{s} \in T(\bar{x})$ if and only if $0 \in \Phi(\bar{s}, \bar{x})$.

(iii) $\bar{x} \in \Omega$ with $\bar{s} \in T(\bar{x})$ if and only if $\Phi(\bar{s}, \bar{x}) \cap C \neq \emptyset$.

Proof. (i) If not, there exists $\tau \in \Phi(\bar{s}, \bar{x}) \cap$ int $C$ for some $\bar{x} \in$ $K$ and $\bar{s} \in T(\bar{x})$. Then $f(\bar{s}, \bar{x}, K) \cap(\tau-\operatorname{int} C)=\emptyset$ and $\tau \in$ int $C$. Hence, $0 \notin f(\bar{s}, \bar{x}, K)$ which contradicts the fact that $0 \in f(s, x, K)$ for all $x \in K$ and for all $s \in T(x)$.

(ii) $0 \in \Phi(\bar{s}, \bar{x})$ if and only if $f(\bar{s}, \bar{x}, K) \cap(-\operatorname{int} C)=\emptyset$ if and only if $\bar{x} \in \Omega$.

(iii) By (i) and (ii), we have $\bar{x} \in \Omega$ with $\bar{s} \in T(\bar{x})$ if and only if $0 \in \Phi(\bar{s}, \bar{x})$ if and only if $\Phi(\bar{s}, \bar{x}) \cap C \neq \emptyset$. 
Definition 2. A sequence $\left\{\left(s_{n}, x_{n}\right) \in Z \times K: s_{n} \in T\left(x_{n}\right)\right\}$ is a minimizing sequence for $\Phi$ if for every neighborhood $U_{Y} \in$ $\mathfrak{N}_{Y}(0)$ of 0 , there is $n_{0} \in \mathbb{N}$, such that $\Phi\left(s_{n}, x_{n}\right) \cap U_{Y} \neq \emptyset$ for all $n \geq n_{0}$.

Definition 3. (GSEP) is $M$-well-posed if it satisfies the following conditions:

(i) there exists at least one solution, that is, the set $\Omega \neq \emptyset$;

(ii) for every minimizing sequence $\left\{\left(s_{n}, x_{n}\right)\right\}$ and for every $U_{X} \in \mathfrak{N}_{X}(0)$, there is $n_{0} \in \mathbb{N}$ such that $x_{n} \in \Omega+U_{X}$ for all $n \geq n_{0}$.

Definition 4. For $\epsilon \in C$, the $\epsilon$-approximate solution set of (GSEP) is defined by $\Omega(\epsilon)=\{x \in K: \Phi(s, x) \cap(C-\epsilon) \neq \emptyset$ for some $s \in T(x)\}$.

We can easily see that $\Omega(0)=\Omega$ in Definition 4 . Indeed, $\bar{x} \in \Omega(0)$ with some $\bar{s} \in T(\bar{x})$ if and only if $\Phi(\bar{s}, \bar{x}) \cap C \neq \emptyset$ if and only if $\bar{x} \in \Omega$ from Lemma 1(iii).

Definition 5. (GSEP) is B-well-posed if it satisfies the following conditions:

(i) there exists at least one solution, that is, the set $\Omega \neq \emptyset$;

(ii) the mapping $\Omega: C \rightrightarrows X$ is upper Hausdorff continuous at $\epsilon=0$; that is, for every $U_{X} \in \mathfrak{N}_{X}(0)$, there exists $U_{Y} \in \mathfrak{N}_{Y}(0)$ such that $\Omega(\epsilon) \subset \Omega+U_{X}$ for every $\epsilon \epsilon$ $U_{Y} \cap C$.

Definition 3 arises from [8], and Definition 5 is originally proposed by [9].

Definition 6 (see $[16,17])$. A set-valued mapping $T: X \rightrightarrows Z$ is

(i) upper semicontinuous if for every $x \in X$ and every open set $V$ in $Y$ with $T(x) \subset V$, there exists a neighborhood $W(x)$ of $x$ such that $T(W(x)) \subset V$;

(ii) lower semicontinuous if for every $x \in X$ and every open neighborhood $V(y)$ of every $y \in T(x)$, there exists a neighborhood $W(x)$ of $x$ such that $T(u) \cap$ $V(y) \neq \emptyset$ for all $u \in W(x)$

(iii) continuous if it is both upper semicontinuous and lower semicontinuous.

We note that $T$ is upper semicontinuous at $x_{0}$ and $T\left(x_{0}\right)$ is compact; then for any net $\left\{x_{v}\right\} \subset X, x_{v} \rightarrow x_{0}$, and for any net $y_{v} \in T\left(x_{v}\right)$ for each $v$, there exists $y_{0} \in T\left(x_{0}\right)$ and a subnet $\left\{y_{v_{\alpha}}\right\}$ such that $y_{v_{\alpha}} \rightarrow y_{0}$. We can refer to [18] for more details. We also note that $T$ is lower semicontinuous at $x_{0}$ if for any net $\left\{x_{\gamma}\right\} \subset X, x_{\gamma} \rightarrow x_{0}, y_{0} \in T\left(x_{0}\right)$ implies that there exists net $y_{v} \in T\left(x_{v}\right)$ such that $y_{v} \rightarrow y_{0}$. For more details, we refer the reader to [16] or [17]. Another more weaker upper semi-continuity is said above $C$-upper Hausdorff semicontinuous [9]. A mapping $T: X \rightrightarrows Z$ is above $C$-upper Hausdorff semicontinuous if for every $x \in X$ and every open set $W_{Y} \in \mathfrak{N}_{Y}(0)$, there exists a neighborhood $V_{X} \in$ $\mathfrak{N}_{X}(0)$ such that $T\left(x+V_{Y}\right) \subset T(x)+W_{Y}-C$. Obviously, the upper Hausdorff continuity is weaker than the upper semi-continuity, and an upper Hausdorff continuous mapping is an above $C$-upper Hausdorff semi-continuous mapping.

\section{2. $B$-Well-Posed and $M$-Well-Posed}

In this section, we will discuss the relationship between these two kinds of well-posedness. The first one is given as follows.

Example 7. (i) There is an example that satisfies $M$-wellposed, but not $B$-well-posed. (ii) There is an example that satisfies both $B$-well-posed and $M$-well-posed.

Solution. (i) The first one is inspired by the example of [10]. Let $X=Z=\mathbb{R}, Y=\mathbb{R}^{2}, C=\mathbb{R}_{+}^{2}, K=\mathbb{R}_{+}, T: K \rightrightarrows Z$ satisfy $T(x)=[x / 2, x]$ for all $x \in K$. The set-valued mapping $f: Z \times K \times K \rightrightarrows Y$ is defined by

$$
\begin{aligned}
& f(s, x, y) \\
& = \begin{cases}\{(0,0)\}, & \text { if } x=0, y \geq 0 ; \\
\{(y-s, 0)\}, & \text { if } x>0,0 \leq y<2 s ; \\
\left\{\left(s,-\frac{8 s}{y^{3}}\right),\left(-s,-\frac{8 s}{y^{3}}\right)\right\}, & \text { otherwise, }\end{cases}
\end{aligned}
$$

for all $(s, x, y) \in Z \times K \times K$ with $s \in T(x)$.

Then the set of all solutions for (GSEP) is $\Omega=\{0\}$. For any $x \in K$ and any $s \in T(x)$, the minimizing mapping is

$$
\Phi(s, x)= \begin{cases}\{(0,0)\} & \text { if } x=0 \\ \left\{\left(\{-s\} \times\left[-\frac{1}{s^{2}}, 0\right]\right) \cup\left\{\left(s,-\frac{1}{s^{2}}\right)\right\}\right\}, & \text { if } x>0\end{cases}
$$

If we choose $U_{X}=(-1 / 2,1 / 2)$, a neighborhood of 0 , and $x_{n}=n, \epsilon_{n}=\left(0,1 / n^{2}\right)$ for all $n \in \mathbb{N}$, we can easily see that $\epsilon_{n} \rightarrow(0,0)$ as $n \rightarrow \infty$, and $n \in \Omega\left(\epsilon_{n}\right) \backslash\left(\Omega+U_{X}\right)$ for all $n \in \mathbb{N}$. Thus, (GSEP) is not $B$-well-posed.

Nevertheless, for any minimizing sequence $\left\{\left(s_{n}, x_{n}\right)\right\}$ for $\Phi$ with $s_{n} \in T\left(x_{n}\right)$ for all $n$ and for every $U_{Y} \in \mathfrak{N}_{Y}(0)$, there exists $n_{0} \in \mathbb{N}$ such that $\Phi\left(s_{n}, x_{n}\right) \cap U_{Y} \neq \emptyset$ for $n \geq n_{0}$. This will force that $x_{n} \rightarrow 0$ as $n \rightarrow \infty$, and hence $x_{n} \in \Omega+U_{X}$ for all $n \geq n_{0}$. Therefore, (GSEP) is $M$-well-posed.

(ii) We modify the above example as follows. Let $X, Z, Y$, $C, K, T$ be given the same as in (i). The set-valued mapping $f: Z \times K \times K \rightrightarrows Y$ is defined by

$$
f(s, x, y)= \begin{cases}\{(0,0)\}, & \text { if } x=0, y \geq 0 \\ \{(y-s, 0)\}, & \text { if } x>0,0 \leq y<2 s \\ \left\{\left(s,-\frac{8 s}{y^{3}}\right)\right\}, & \text { otherwise, }\end{cases}
$$

for all $(s, x, y) \in Z \times K \times K$ with $s \in T(x)$. 
Then the set of all solutions for (GSEP) is $\Omega=K$. For any $x \in K$ and any $s \in T(x)$, the minimizing mapping is

$$
\Phi(s, x)= \begin{cases}\{(0,0)\} & \text { if } x=0 \\ \left\{\left(s,-\frac{1}{s^{2}}\right)\right\}, & \text { if } x>0 .\end{cases}
$$

Since for any minimizing sequence $\left\{\left(s_{n}, x_{n}\right)\right\}$, and for every $U_{X} \in \mathfrak{N}_{X}(0)$, we always have $x_{n} \in \Omega+U_{X}$. Thus, (GSEP) is $M$-well-posed. Furthermore, since $\Omega+U_{X}=K+$ $U_{X}$, for all $\epsilon \in \mathbb{R}_{+}^{2}$, we always have $\Omega(\epsilon) \subset \Omega+U_{X}$. Hence, (GSEP) is $B$-well-posed.

From the above observation, the $M$-well-posed is weaker than $B$-well-posed for (GSEP). What conditions need to be added so that the converse statement can be valid? The following results will be one of the answers.

Proposition 8. (a) If (GSEP) is B-well-posed, then it is $M$ well-posed. (b) If (GSEP) is $M$-well-posed, and for every $W_{Y} \in$ $\mathfrak{N}_{Y}(0)$, there exists $U_{Y} \in \mathfrak{N}_{Y}(0)$ such that

$$
\Phi(h(K \backslash \mathrm{cl}(\Omega))) \cap\left(C+U_{Y}\right) \subset W_{Y},
$$

where $h(x)=T(x) \times\{x\}$ for all $x \in K \backslash \mathrm{cl}(\Omega)$, then $(G S E P)$ is $B$-well-posed.

Proof. For the idea of the proof, we can use the similar direction of [10, Propositions 3 and 4]. For the sake of clarity, we give the proof of (b) as follows. Suppose that (GSEP) is not $B$ well-posed. Then there is a neighborhood $\bar{U}_{X}$ of 0 , and sequences $\left\{\epsilon_{n}\right\} \subset C$ with $\epsilon_{n} \rightarrow 0$ and $x_{n} \in \Omega\left(\epsilon_{n}\right)$ such that

$$
x_{n} \notin \Omega+\bar{U}_{X}, \quad \forall n \in \mathbb{N} \text {. }
$$

This means

$$
x_{n} \notin \mathrm{cl}(\Omega), \quad \forall n \in \mathbb{N} .
$$

Since $x_{n} \in \Omega\left(\epsilon_{n}\right)$, there exists $s_{n} \in T\left(x_{n}\right)$ such that

$$
\Phi\left(s_{n}, x_{n}\right) \cap\left(C-\epsilon_{n}\right) \neq \emptyset, \quad \forall n \in \mathbb{N} .
$$

Now, we separate into two cases.

Case 1. If the sequence $\left\{\left(s_{n}, x_{n}\right)\right\}$ is a minimizing sequence, then by $M$-well-posedness, for this $\bar{U}_{X}$, there is a $n_{0} \in \mathbb{N}$ such that $x_{n} \in \Omega+\bar{U}_{X}$, for all $n \geq n_{0}$, which contradicts (8).

Case 2. If the sequence $\left\{\left(s_{n}, x_{n}\right)\right\}$ is not a minimizing sequence, then there is a $W_{Y} \in \mathfrak{N}_{Y}(0)$ and a subsequence $\left\{x_{n_{k}}\right\}$ of $\left\{x_{n}\right\}$ with a corresponding subsequence $\left\{s_{n_{k}}\right\} \in T\left(\left\{x_{n_{k}}\right\}\right)$ such that

$$
\Phi\left(s_{n_{k}}, x_{n_{k}}\right) \cap W_{Y}=\emptyset, \quad \forall k \in \mathbb{N} .
$$

By relation (10), we have $\Phi\left(s_{n_{k}}, x_{n_{k}}\right) \cap\left(C-\epsilon_{n_{k}}\right) \neq \emptyset$, for all $k \in \mathbb{N}$. For this $W_{Y}$ and condition (7), there is a symmetric neighborhood $U_{Y} \in \mathfrak{N}_{Y}(0)$ such that $\Phi\left(s_{n_{k}}, x_{n_{k}}\right) \cap\left(C+U_{Y}\right) \subset$ $W_{Y}$, for all $k \in \mathbb{N}$. For $k$ large enough, $-\epsilon_{n_{k}} \in U_{Y}$. Taking $\eta_{n_{k}} \in \Phi\left(s_{n_{k}}, x_{n_{k}}\right) \cap\left(C+U_{Y}\right)$ for all $k \in \mathbb{N}$. This implies that, for $k$ large enough, $\eta_{n_{k}} \in \Phi\left(s_{n_{k}}, x_{n_{k}}\right) \cap W_{Y}$ which contradicts (11). This completes the proof.
We need the following lemma for the next criterion for $M$-well-posedness of (GSEP).

Lemma 9. Let $Y$ be a regular topological vector spaces, and let $A$ be a nonempty compact subset of $Y$. Suppose that $A \cap(-C)=$ $\emptyset$; then there is a neighborhood $W_{Y}$ of 0 such that $\left(A+W_{Y}\right) \cap$ $\left(W_{Y}-C\right)=\emptyset$. In particular, $\left(A+W_{Y}\right) \cap W_{Y}=\emptyset$.

Proof. Suppose that $A \cap(-C)=\emptyset$. For all $\eta \in A$, we have $\eta \notin-C$. Since $Y$ is regular, there is a neighborhood $U_{Y}^{\eta}$ of 0 such that

$$
\left(\eta+U_{Y}^{\eta}\right) \bigcap\left(U_{Y}^{\eta}-C\right)=\emptyset .
$$

Since $A$ is a nonempty compact subset, the set

$$
A \subset \bigcup_{\eta \in A}\left(\eta+U_{Y}^{\eta}\right) \text {. }
$$

There exist $\eta_{1}, \eta_{2}, \ldots, \eta_{n} \in A$, such that

$$
A \subset \bigcup_{i=1}^{n}\left(\eta_{i}+U_{Y}^{\eta_{i}}\right) \subset A+\bigcup_{i=1}^{n} U_{Y}^{\eta_{i}} .
$$

Let $W_{Y}=\bigcap_{i=1}^{n} U_{Y}^{\eta_{i}}$. Then

$$
\left(A+\bigcup_{i=1}^{n} U_{Y}^{\eta_{i}}\right) \bigcap\left(W_{Y}-C\right)=\emptyset .
$$

Since $W_{Y} \subset \bigcup_{i=1}^{n} U_{Y}^{\eta_{i}}$, we have

$$
\left(A+W_{Y}\right) \cap\left(W_{Y}-C\right)=\emptyset .
$$

We note that, although every compact regular space is a normal space, Lemma 9 is not so intuitive. Furthermore, if the set $A$ is not compact, the conclusion may not hold. For example, we choose $A=\left\{(x, y) \in \mathbb{R}^{2}: y \geq x^{-2}, x<0\right\}$ and $C=\mathbb{R}_{+}^{2}$.

Now, we present first criterion of $M$-well-posedness for (GSEP).

Theorem 10. Let $X, Y, Z$ be three Hausdorff topological vector spaces where $X$ is a finite dimensional space and $Y$ is regular, let $K$ be a nonempty closed convex subset of $X$, and let $C \subset Y$ be a closed convex and pointed cone with apex at the origin and $\operatorname{int} C \neq \emptyset$. The mapping $f: Z \times K \times K \rightrightarrows Y$ is upper semi-continuous with nonempty compact values and satisfies $f(s, x, x)=\{0\}$ for all $x \in K$ and for all $s \in T(x)$, and the mapping $T: K \rightrightarrows Z$ is upper semi-continuous with nonempty compact values, such that

(i) the solution set $\Omega$ of (GSEP) is nonempty and bounded;

(ii) the minimizing mapping $\Phi$ is upper Hausdorff continuous on $T(K) \times K$;

(iii) $f(s, x, y) \cap(-C)=\emptyset$ for all $x \in \Omega$, for all $s \in T(K)$ and for all $y \in K \backslash \Omega$; 
(iv) the mapping $(s, x) \rightarrow f(s, x, y)$ is above-C-upper Hausdorf continuous on $Z \times K$ for every $y \in K$, and the mapping $x \rightarrow f(s, x, y)$ is above-C-concave [19] on $K$ for every $s \in T(K)$ and $y \in K$;

(v) for every minimizing sequence $\left\{\left(s_{n}, x_{n}\right)\right\} \subset T(K) \times K$, and for each $(s, y) \in T(K) \times K$, there is a sequence $\left\{\zeta_{n}\right\}$ with $\zeta_{n} \in f\left(s, x_{n}, y\right)$ for each $n \in \mathbb{N}$ is a bounded sequence in $Y$.

\section{Then (GSEP) is M-well-posedness.}

Proof. We prove it by contradiction. Suppose that (GSEP) is not $M$-well-posedness. Then there exists a minimizing sequence $\left\{\left(s_{n}, x_{n}\right)\right\} \subset T K \times K$ and $\epsilon>0$ such that

$$
x_{n} \notin \Omega+\epsilon B,
$$

for infinitely many $n$, where $B$ denotes the unit open ball in $X$. Let us choose a subsequence from $\left\{\left(s_{n}, x_{n}\right)\right\}$ so that the relation (17) holds for all elements of the subsequence. Such a subsequence is still a minimizing sequence, and we still denote it by $\left\{\left(s_{n}, x_{n}\right)\right\}$ if there is no any confusion. Now, we separate our discussion into two cases.

Case 1. If the sequence $\left\{x_{n}\right\}$ is bounded, then it has a convergent subsequence $\left\{x_{n_{k}}\right\}$ that converges to some point $x^{\star} \in X$ with a corresponding subsequence $\left\{s_{n_{k}}\right\}$ with $s_{n_{k}} \in T\left(x_{n_{k}}\right)$ for every $k \in \mathbb{N}$. By the upper semi-continuity of $T$, there exists a convergent subsequence of $\left\{s_{n_{k}}\right\}$ (without any confuse, we still denote it by $\left.\left\{s_{n_{k}}\right\}\right)$ converges to some point $s^{\star} \in T\left(x^{\star}\right)$. From (17), $x_{n_{k}} \notin \Omega+\epsilon B$ for every $k \in \mathbb{N}$. Hence, $x^{\star} \notin \Omega$, and by Lemma 1, we have $0 \notin \Phi\left(s^{\star}, x^{\star}\right)$. By Lemma 9, there is a neighborhood $U_{Y} \in \mathfrak{N}_{Y}(0)$ such that

$$
\left(\Phi\left(s^{\star}, x^{\star}\right)+W_{Y}\right) \cap W_{Y}=\emptyset
$$

Since $\left\{\left(s_{n_{k}}, x_{n_{k}}\right)\right\}$ is a minimizing sequence, for each $k$, we can choose $\eta_{n_{k}} \in \Phi\left(s_{n_{k}}, x_{n_{k}}\right)$ such that $\eta_{n_{k}} \rightarrow 0$. Since $\Phi$ is upper Hausdorff continuity of $\Phi$ at $\left(s^{\star}, x^{\star}\right)$, we have

$$
\Phi\left(s_{n_{k}}, x_{n_{k}}\right) \subset \Phi\left(s^{\star}, x^{\star}\right)+W_{Y}
$$

Hence, for $k$ large enough, $\eta_{n_{k}} \in\left(\Phi\left(s^{\star}, x^{\star}\right)+W_{Y}\right) \cap W_{Y}$ which contradicts (18).

Case 2. If the sequence $\left\{x_{n}\right\}$ is unbounded. Since $\Omega$ is bounded, so are $\Omega+\epsilon B$ and $\operatorname{cl}(\Omega+\epsilon B)$. Then the set $\operatorname{cl}(\Omega+\epsilon B)$ is compact. We denote that $\Omega \cap \partial(\Omega+\epsilon B)=\emptyset$, where $\partial(\Omega+\epsilon B)$ means the boundary of $\Omega+\epsilon B$. Since the sequence $\left\{x_{n}\right\}$ is unbounded, there is a subsequence $\left\{x_{n_{k}}\right\}$ with $\left\|x_{n_{k}}\right\| \rightarrow \infty$ as $k \rightarrow \infty$. Without loss of generality, we may assume that $x_{n} \notin \partial(\Omega+\epsilon B)$ for all $n \in \mathbb{N}$. Fix any $\bar{x} \in \Omega$ and let $v_{n}=\lambda_{n} \bar{x}+\left(1-\lambda_{n}\right) x_{n} \in \partial(\Omega+\epsilon B)$ for any $n \in \mathbb{N}$, where $\lambda_{n}=\sup \left\{\lambda \in[0,1]: \lambda \bar{x}+(1-\lambda) x_{n} \notin \Omega+\epsilon B\right\}$. After a simple calculation, we can see that $\lambda_{n} \rightarrow 1$ as $n \rightarrow \infty$. Hence, $\left\{v_{n}\right\}$ has a subsequence $\left\{v_{n_{k}}\right\}$ that converges to some point $v^{\star} \in \partial(\Omega+\epsilon B)$. For the similar process in Case 1, we have a subsequence $\left\{t_{n_{k}}\right\}$ of the corresponding sequence $\left\{t_{n}\right\}$ with $t_{n} \in T\left(v_{n}\right)$ converges to some point $t^{\star} \in T\left(v^{\star}\right)$. By the above $C$-concavity of $f$ in $x$, we have

$$
\begin{gathered}
\lambda_{n_{k}} f\left(t_{n_{k}}, \bar{x}, v^{\star}\right)+\left(1-\lambda_{n_{k}}\right) f\left(t_{n_{k}}, x_{n_{k}}, v^{\star}\right) \\
\subset f\left(t_{n_{k}}, v_{n_{k}}, v^{\star}\right)-C .
\end{gathered}
$$

By condition (v), there is a bounded sequence $\left\{\zeta_{n_{k}}\right\}$ with $\zeta_{n_{k}} \in$ $f\left(t_{n_{k}}, x_{n_{k}}, v^{\star}\right)$ for each $k \in \mathbb{N}$ in $Y$. Hence, by (20), we have

$$
\lambda_{n_{k}} f\left(t_{n_{k}}, \bar{x}, v^{\star}\right)+\left(1-\lambda_{n_{k}}\right) \zeta_{n_{k}} \subset f\left(t_{n_{k}}, v_{n_{k}}, v^{\star}\right)-C .
$$

Next, we claim that

$$
f\left(t^{\star}, \bar{x}, v^{\star}\right) \cap(-C) \neq \emptyset .
$$

Indeed, if $f\left(t^{\star}, \bar{x}, v^{\star}\right) \cap(-C)=\emptyset$. By Lemma 9, there is a neighborhood $U_{Y} \in \mathfrak{N}_{Y}(0)$ such that

$$
\left(f\left(t^{\star}, \bar{x}, v^{\star}\right)+U_{Y}\right) \cap\left(U_{Y}-C\right)=\emptyset .
$$

For this $U_{Y}$, by the above $C$-upper Hausdorff continuity of $f$ and the fact that $f\left(t^{\star}, v^{\star}, v^{\star}\right)=\{0\}$, we have

$$
\begin{gathered}
f\left(t_{n_{k}}, v_{n_{k}}, v^{\star}\right) \subset U_{Y}-C, \\
f\left(t_{n_{k}}, \bar{x}, v^{\star}\right) \subset f\left(t^{\star}, \bar{x}, v^{\star}\right)+U_{Y}
\end{gathered}
$$

for $k$ large enough. Since the sequence $\left\{\zeta_{n_{k}}\right\}$ is bounded, the left-hand side of (21) will fell into $f\left(t^{\star}, \bar{x}, v^{\star}\right)+U_{Y}$ for $k$ large enough. But in this situation, we can see that $\left(f\left(t^{\star}, \bar{x}, v^{\star}\right)+\right.$ $\left.U_{Y}\right) \cap\left(U_{Y}-C\right) \neq \emptyset$ which contradicts (23). Thus, the relation (22) holds. Since $\bar{x} \in \Omega$ and $t^{\star} \in T(K)$, by condition (iii) we have $v^{\star} \in \Omega$ which contradicts the fact that $v^{\star} \in \partial(\Omega+\epsilon B)$.

From the discussions of above two cases, (GSEP) is $M$ well-posedness.

Remark 11. Theorem 10 generalize the Theorem 1 of [10] to (GSEP).

Lemma 12. Suppose that $F: K \rightrightarrows Y$ and $f: K \times K \rightrightarrows Y$ satisfy $f(x, y)=F(y)-F(x)$ for all $x, y \in K$, then

$$
\operatorname{Min}_{w} f(x, K) \subset \operatorname{Min}_{w} F(K)-F(x)
$$

for all $x \in K$.

Proof. For any fixed $x \in K$. Choose any $\xi \in \operatorname{Min}_{w} f(x, K)$; we have $\xi \in F(K)-F(x)$ and $(F(K)-F(x)) \cap(\xi-\operatorname{int} C)=\emptyset$. There exist $\xi_{1} \in F(K)$ and $\xi_{2} \in F(x)$ such that $\xi=\xi_{1}-\xi_{2}$ and $(F(K)-F(x)) \cap\left(\xi_{1}-\xi_{2}-\operatorname{int} C\right)=\emptyset$. That is, $\left(F(K)+\xi_{2}-\right.$ $F(x)) \cap\left(\xi_{1}-\right.$ int $\left.C\right)=\emptyset$. Since $0 \in \xi_{2}-F(x)$ and $F(K) \subset F(K)+$ $\xi_{2}-F(x)$, we know that $F(K) \cap\left(\xi_{1}-\operatorname{int} C\right)=\emptyset$. Thus, $\xi_{1} \in$ $\operatorname{Min}_{w} F(K)$, and hence, $\xi \in \operatorname{Min}_{w} F(K)-F(x)$. Therefore,

$$
\operatorname{Min}_{w} f(x, K) \subset \operatorname{Min}_{w} F(K)-F(x) .
$$


Lemma 13. Let $X, Y, C, K$ be given the same as in Theorem 10, let $f$ be as given in Lemma 12, and let $F: K \rightrightarrows Y$ be upper semi-continuous with nonempty compact values such that the set $\Omega=w-\operatorname{Eff}(F, K)$ is bounded, where $w-\operatorname{Eff}(F, K)=$ $\{x \in K: y \in F(x), F(K) \cap(y-\operatorname{int} C)=\emptyset\}$. Any sequence $\left\{x_{n}\right\}$ satisfies for every neighborhood $U_{Y} \in \mathfrak{N}_{Y}(0)$ of 0 ; there is $n_{0} \in \mathbb{N}$, such that $\operatorname{Min}_{w} f\left(x_{n}, K\right) \cap U_{Y} \neq \emptyset$ for all $n \geq n_{0}$. Then there exists a bounded sequence $\left\{\eta_{n}\right\}$ in $Y$ with $\eta_{n} \in F\left(x_{n}\right)$, for all $n \in \mathbb{N}$.

Proof. Since $\Omega$ is bounded, its closure $\mathrm{cl}(\Omega)$ is compact. By the upper semi-continuity of $F, F(\operatorname{cl}(\Omega))$ is compact. Hence it is bounded, so is $F(\Omega)$. Fixed $W_{Y} \in \mathfrak{N}_{Y}(0)$, a symmetric neighborhood of 0 . Since the sequence $\left\{x_{n}\right\}$ satisfies for every neighborhood $U_{Y} \in \mathfrak{N}_{Y}(0)$ of 0 , there is $n_{0} \in \mathbb{N}$, such that $\operatorname{Min}_{w} f\left(x_{n}, K\right) \cap U_{Y} \neq \emptyset$ for all $n \geq n_{0}$. From Lemma 12, we have

$$
\left(\operatorname{Min}_{w} F(K)-F\left(x_{n}\right)\right) \cap W_{Y} \neq \emptyset
$$

for all $n \geq n_{0}$. We can pick some points $\xi_{n} \in \operatorname{Min}_{w} F(K)$, and $\eta_{n} \in F\left(x_{n}\right)$ such that

$$
\xi_{n}-\eta_{n} \in W_{Y}
$$

for all $n \geq n_{0}$. Since $W_{Y}$ is symmetric, we have

$$
\begin{aligned}
\eta_{n} & \in \xi_{n}+W_{Y} \subset \operatorname{Min}_{w} F(K)+W_{Y} \\
& =F(w-\operatorname{Eff}(F, K))+W_{Y} \\
& =F(\Omega)+W_{Y}
\end{aligned}
$$

for all $n \geq n_{0}$. Since $F(\Omega)$ is bounded, so is $F(\Omega)+W_{Y}$. Therefore, the sequence $\left\{\eta_{n}\right\}$ is bounded.

Now, let us present another criterion for $M$-wellposedness of (GSEP).

Theorem 14. Let $X, Y, Z, C, K, f, T$ be given the same as in Theorem 10. Suppose that

(i) the solution set $\Omega$ of (GSEP) is nonempty and compact;

(ii) for every $x, z \in K$ and $s \in T(K)$, if $f(s, x, z) \cap C \neq \emptyset$, then $f(s, z, x) \cap(-C) \neq \emptyset$;

(iii) $f(s, x, z) \cap(-C)=\emptyset$ for all $x \in \Omega$, for all $s \in T(K)$ and for all $z \in K \backslash \Omega$;

(iv) the mapping $(s, x) \rightarrow f(s, x, y)$ is above $C$-upper Hausdorff continuous on $Z \times K$ for every $y \in K$, and the mapping $x \rightarrow f(s, x, y)$ is above $C$-concave on $K$ for every $\in \in T(K)$ and $y \in K$;

(v) for each minimizing sequence $\left\{\left(s_{n}, x_{n}\right)\right\} \subset T(K) \times K$, and for each $(y, s) \in K \times Z$, there is a sequence $\left\{\zeta_{n}\right\}$ with $\zeta_{n} \in f\left(s, x_{n}, y\right)$ for each $n \in \mathbb{N}$ is a bounded sequence in $Y$;

(vi) for every $x \in K \backslash \Omega$ and for every $s \in T(x)$, $\operatorname{Min}_{w} f(s, x, K) \subset f(s, x, \Omega)$.

Then (GSEP) is M-well-posedness.
Proof. We prove it by contradiction. Suppose that (GSEP) is not $M$-well-posedness. Hence there exists a minimizing sequence $\left\{\left(s_{n}, x_{n}\right)\right\} \subset T(K) \times K$ and $\epsilon>0$ such that the relation (17) holds. If the sequence $\left\{x_{n}\right\}$ is unbounded, by a similar process in Case 2 of Theorem 10 we know that (GSEP) is $M$-well-posedness. If the sequence $\left\{x_{n}\right\}$ is bounded, then it has a convergent subsequence that converges to some point $x^{\star} \in X$ with a corresponding subsequence $\left\{s_{n}\right\}$ with $s_{n} \in$ $T\left(x_{n}\right)$ for every $n \in \mathbb{N}$. We still denote it by $\left\{x_{n}\right\}$ if there is no confusion. The relation (17) tells us that

$$
x^{\star} \notin \Omega \text {. }
$$

Since $\left\{\left(s_{n}, x_{n}\right)\right\}$ is a minimizing sequence, we can choose a sequence $\left\{\tau_{n}\right\}$ with $\tau_{n} \rightarrow 0$, where $\tau_{n} \in \Phi\left(x_{n}\right)=$ $\operatorname{Min}_{w} f\left(s_{n}, x_{n}, K\right)$ for all $n \in \mathbb{N}$. For the same process as in Case 1 of Theorem 10, by the upper semi-continuity of $T$, there exists a convergent subsequence of $\left\{s_{n}\right\}$ (without any confusion, we still denote it by $\left\{s_{n}\right\}$ ) that converges to some point $s^{\star} \in T\left(x^{\star}\right)$. Since $x_{n} \in K \backslash \Omega$, by condition (vi), $\tau_{n} \in$ $\operatorname{Min}_{w} f\left(s_{n}, x_{n}, K\right) \subset f\left(s_{n}, x_{n}, \Omega\right)$. Then, for each $n \in \mathbb{N}$, there is a $z_{n} \in \Omega$ such that $\tau_{n} \in f\left(s_{n}, x_{n}, z_{n}\right)$. Since $\Omega$ is compact, there is a subsequence $\left\{z_{n_{k}}\right\}$ of $\left\{z_{n}\right\}$ that converges to some point $z^{\star} \in \Omega$. Now we claim that $f\left(s^{\star}, x^{\star}, z^{\star}\right) \cap C \neq \emptyset$. Indeed, suppose that $f\left(s^{\star}, x^{\star}, z^{\star}\right) \cap C=\emptyset$. By Lemma 9, there is $W_{Y} \in \mathbb{N}_{Y}(0)$ such that

$$
\left(f\left(s^{\star}, x^{\star}, z^{\star}\right)+W_{Y}\right) \cap\left(C+W_{Y}\right)=\emptyset,
$$

or

$$
\left(f\left(s^{\star}, x^{\star}, z^{\star}\right)+W_{Y}-C\right) \cap W_{Y}=\emptyset .
$$

By the above $C$-upper Hausdorff continuity of $f$, for this $W_{Y}$, there is $k_{0} \in \mathbb{N}$ such that

$$
\tau_{n_{k}} \in f\left(s_{n_{k}}, x_{n_{k}}, z_{n_{k}}\right) \subset f\left(s^{\star}, x^{\star}, z^{\star}\right)+W_{Y}-C,
$$

for all $k \geq k_{0}$. Thus, from (32), $\tau_{n_{k}} \notin W_{Y}$ which contradicts the fact that $\tau_{n_{k}} \rightarrow 0$. Hence, $f\left(s^{\star}, x^{\star}, z^{\star}\right) \cap C \neq \emptyset$. By condition (ii), $f\left(s^{\star}, z^{\star}, x^{\star}\right) \cap(-C) \neq \emptyset$. Since $z^{\star} \in \Omega$, by condition (iii), $x^{\star} \in \Omega$ which contradicts (30). Hence (GSEP) is $M$-wellposedness.

Let us present the third criterion for $M$-well-posedness of (GSEP) as follows.

Theorem 15. Let $X, Y, Z, C, K, T, f$ be given the same as in Theorem 10. Suppose that

(i) the solution set $\Omega$ of (GSEP) is nonempty and bounded;

(ii) $f(s, x, z) \cap(-C)=\emptyset$ and $f(s, z, x) \cap(-C)=\emptyset$ for all $z \in \operatorname{cl}(\Omega)$, for all $s \in T(K)$ and for all $x \in K \backslash \operatorname{cl}(\Omega)$;

(iii) the mapping $x \rightarrow f(s, x, y)$ is above $C$-concave on $K$ for every $(s, y) \in T(K) \times K$, and the mapping $s \rightarrow$ $f(s, x, y)$ is lower semi-continuous on $T(K)$, for every $(x, y) \in K \times K$;

(iv) for every $x \in K \backslash \mathrm{cl}(\Omega)$ and for every $s \in T(x)$, $\operatorname{Min}_{w} f(s, x, K) \subset f(s, x, \operatorname{cl}(\Omega))$.

Then (GSEP) is M-well-posedness. 
Proof. Suppose that (GSEP) is not $M$-well-posedness. Then we have a minimizing sequence $\left\{\left(s_{n}, x_{n}\right)\right\} \subset T(K) \times K$ and $\epsilon>0$ such that the relation (17) holds for all $n \in \mathbb{N}$. We can use the same process as in the proof of Theorem 10 under the situation when we replace $\Omega$ by $\operatorname{cl}(\Omega)$. If the sequence $\left\{x_{n}\right\}$ is unbounded, combining Lemmas 12 and 13, we have the sequences $\left\{x_{n_{k}}\right\},\left\{v_{n}\right\},\left\{v_{n_{k}}\right\},\left\{\lambda_{n}\right\},\left\{t_{n}\right\},\left\{t_{n_{k}}\right\},\left\{\zeta_{n_{k}}\right\}$, and points $\bar{x}, v^{\star}, t^{\star}$ with the same properties as in the proof (Case 2) of Theorem 10. By condition (iii), we have the mapping $x \rightarrow$ $f(s, x, y)$ which is above $C$-concave on $K$ for every $(s, y) \in$ $T(K) \times K$ and the relations (20) and (21) hold. Since the mapping $s \rightarrow f(s, x, y)$ is lower semi-continuous on $T(K)$ for every $(x, y) \in K \times K$ and fix any $\xi \in f\left(t^{\star}, \bar{x}, v^{\star}\right)$, there exists $\xi_{n_{k}} \in f\left(t_{n_{k}}, \bar{x}, v^{\star}\right)$ such that $\xi_{n_{k}} \rightarrow \xi$. For this $\xi_{n_{k}}$, by (21), there exists $v_{n_{k}} \in f\left(t_{n_{k}}, v_{n_{k}}, v^{\star}\right)$ such that

$$
\lambda_{n_{k}} \xi_{n_{k}}+\left(1-\lambda_{n_{k}}\right) \zeta_{n_{k}} \in v_{n_{k}}-C \text {. }
$$

Since the mapping $(s, x) \rightarrow f(s, x, y)$ is upper semi-continuous, hence it is upper Hausdorff continuous on $Z \times K$ for every $y \in K$, and $\left(t_{n_{k}}, v_{n_{k}}\right) \rightarrow\left(t^{\star}, \bar{x}\right)$, for any given $W_{Y} \in \mathfrak{N}_{Y}(0)$

$$
f\left(t_{n_{k}}, v_{n_{k}}, v^{\star}\right) \subset f\left(t^{\star}, v^{\star}, v^{\star}\right)+W_{Y}=W_{Y}
$$

for $k$ large enough. From (34) and (35) and the fact that $\left\{\lambda_{n_{k}}\right\} \rightarrow 1$, we have

$$
\xi \in W_{Y}-C
$$

Next, we claim that

$$
f\left(t^{\star}, \bar{x}, v^{\star}\right) \cap(-C) \neq \emptyset .
$$

Indeed, if not, $f\left(t^{\star}, \bar{x}, v^{\star}\right) \cap(-C)=\emptyset$. By Lemma 9, there exists $N_{Y} \in \mathfrak{N}_{Y}(0)$ such that $\left(f\left(t^{\star}, \bar{x}, v^{\star}\right)+N_{Y}\right) \cap\left(N_{Y}-C\right)=$ $\emptyset$. But this contradicts (36), and hence (37) holds. Since $\bar{x} \in$ $\mathrm{cl}(\Omega)$, by condition (ii), $v^{\star} \in \mathrm{cl}(\Omega)$ which contradicts the fact $v^{\star} \in \partial(\mathrm{cl}(\Omega)+\epsilon B)$. Hence, (GSEP) is $M$-well-posed. On the other hand, if the sequence $\left\{x_{n}\right\}$ is bounded, the sequences $\left\{x_{n_{k}}\right\},\left\{s_{n}\right\},\left\{s_{n_{k}}\right\}$, the points $x^{\star}, s^{\star}$, and the number $\epsilon$ are the same as in the Case 1 of Theorem 10 , so that $x_{n_{k}} \notin \mathrm{cl}(\Omega)+\epsilon B$ for all $k \in \mathbb{N}$. Hence $x^{\star} \notin \operatorname{cl}(\Omega)$. Since $\left\{\left(s_{n}, x_{n}\right)\right\}$ is minimizing, we can choose a sequence $\eta_{n} \in \Phi\left(s_{n}, x_{n}\right)=\operatorname{Min}_{w} f\left(s_{n}, x_{n}, K\right)$ with $\eta_{n} \rightarrow 0$. By condition (v), $\eta_{n} \in \operatorname{Min}_{w} f\left(s_{n}, x_{n}, K\right) \subset$ $f\left(s_{n}, x_{n}, \operatorname{cl}(\Omega)\right)$. For each $n \in \mathbb{N}$, there is $z_{n} \in \mathrm{cl}(\Omega)$ such that $\eta_{n} \in f\left(s_{n}, x_{n}, z_{n}\right)$. Since $\operatorname{cl}(\Omega)$ is compact, there is a subsequence $\left\{z_{n_{k}}\right\}$ of $\left\{z_{n}\right\}$ that converges to some point $z^{\star} \in$ $\operatorname{cl}(\Omega)$. Since $f: Z \times K \times K \rightrightarrows Y$ is upper semi-continuous with nonempty compact values, we have

$$
0 \in f\left(s^{\star}, x^{\star}, z^{\star}\right) \text {. }
$$

From condition (ii) and the fact that $z^{\star} \in \operatorname{cl}(\Omega)$, we have $x^{\star} \in$ $\Omega$ which contradicts the fact $x^{\star} \notin \Omega$. Hence, (GSEP) is $M$ well-posed.

Example 7 tells us that if (GSEP) is $M$-well-posed, then it is $B$-well-posed. But the converse is not true. Proposition 8 proposes a possible condition so that the converse holds. To the end, we state this result as follows.
Corollary 16. Under the framework of Theorem 14 (resp., Theorem 15) the following condition (A) holds:

(A) for every $W_{Y} \in \mathfrak{N}_{Y}(0)$, there is a $U_{Y} \in \mathfrak{N}(0)$ such that

$$
f(g(K \backslash \mathrm{cl}(\Omega))) \cap\left(C+U_{Y}\right) \subset W_{Y},
$$

where $g(x)=h(x) \times \mathrm{cl}(\Omega)$ and $h$ is given in (7).

Then (GSEP) is B-well-posed.

Proof. From Theorem 14 (resp., Theorem 15), (GSEP) is $M$ well-posed. By condition (vi) of Theorem 14 (resp., condition (iv) of Theorem 15), we have

$$
\Phi(s, x) \subset f(s, x, \mathrm{cl}(\Omega))
$$

for every $s \in T(x)$ and $x \in K \backslash \operatorname{cl}(\Omega)$. Hence,

$$
\Phi(h(K \backslash \mathrm{cl}(\Omega))) \subset f(g(K \backslash \mathrm{cl}(\Omega))) .
$$

Combining this with condition (A), the condition (7) holds. Hence, by Proposition 8, (GSEP) is B-well-posed.

\section{Acknowledgments}

This work was supported by Grant no. NSC101-2115-M-039001 of the National Science Council of Taiwan which is gratefully acknowledged. The author would like to thank the referees for their useful comments which have helped to improve some results and the presentation of the paper.

\section{References}

[1] A. N. Tihonov, "Stability of a problem of optimization of functionals," Akademija Nauk SSSR, vol. 6, pp. 631-634, 1966.

[2] L. C. Ceng and Y. C. Lin, "Metric characterizations of $\alpha$-wellposedness for a system of mixed quasivariational-like inequalities in Banach spaces," Journal of Applied Mathematics, vol. 2012, Article ID 264721, 22 pages, 2012.

[3] A. L. Dontchev and T. Zolezzi, Well-Posed Optimization Problems, Springer, Berlin, Germany, 1993.

[4] E. Bednarczuk and J.-P. Penot, "Metrically well-set minimization problems," Applied Mathematics and Optimization, vol. 26, no. 3, pp. 273-285, 1992.

[5] G. P. Crespi, A. Guerraggio, and M. Rocca, "Well posedness in vector optimization problems and vector variational inequalities," Journal of Optimization Theory and Applications, vol. 132, no. 1, pp. 213-226, 2007.

[6] M. Furi and A. Vignoli, "About well-posed minimization problems for functionals in metric spaces," Journal of Optimization Theory and Applications, vol. 5, no. 3, pp. 225-229, 1970.

[7] T. Zolezzi, "Well-posedness criteria in optimization with application to the calculus of variations," Nonlinear Analysis:Theory, Methods \& Applications, vol. 25, no. 5, pp. 437-453, 1995.

[8] E. Miglierina and E. Molho, "Well-posedness and convexity in vector optimization," Mathematical Methods of Operations Research, vol. 58, no. 3, pp. 375-385, 2003.

[9] E. Bednarczuk, "An approach to well-posedness in vector optimization: consequences to stability," Control and Cybernetics, vol. 23, no. 1-2, pp. 107-122, 1994. 
[10] M. Bianchi, G. Kassay, and R. Pini, "Well-posedness for vector equilibrium problems," Mathematical Methods of Operations Research, vol. 70, no. 1, pp. 171-182, 2009.

[11] L. C. Ceng, N. Hadjisavvas, S. Schaible, and J. C. Yao, "Wellposedness for mixed quasivariational-like inequalities," Journal of Optimization Theory and Applications, vol. 139, no. 1, pp. 109$125,2008$.

[12] L. C. Ceng and J. C. Yao, "Well-posedness of generalized mixed variational inequalities, inclusion problems and fixed-point problems," Nonlinear Analysis: Theory, Methods \& Applications, vol. 69, no. 12, pp. 4585-4603, 2008.

[13] Y.-P. Fang, N.-J. Huang, and J.-C. Yao, "Well-posedness of mixed variational inequalities, inclusion problems and fixed point problems," Journal of Global Optimization, vol. 41, no. 1, pp. 117133, 2008.

[14] K. Kimura, Y.-C. Liou, S.-Y. Wu, and J.-C. Yao, "Well-posedness for parametric vector equilibrium problems with applications," Journal of Industrial and Management Optimization, vol. 4, no. 2, pp. 313-327, 2008.

[15] L. Q. Anh, P. Q. Khanh, D. T. M. van, and J.-C. Yao, "Well-posedness for vector quasiequilibria," Taiwanese Journal of Mathematics, vol. 13, no. 2B, pp. 713-737, 2009.

[16] C. Berge, Topological Spaces, Macmillan, New York, NY, USA, 1963.

[17] J.-P. Aubin and A. Cellina, Differential Inclusions, Springer, Berlin, Germany, 1984.

[18] F. Ferro, "Optimization and stability results through cone lower semicontinuity," Set-Valued Analysis, vol. 5, no. 4, pp. 365-375, 1997.

[19] Y.-C. Lin, Q. H. Ansari, and H.-C. Lai, "Minimax theorems for set-valued mappings under cone-convexities," Abstract and Applied Analysis, vol. 2012, Article ID 310818, 26 pages, 2012. 


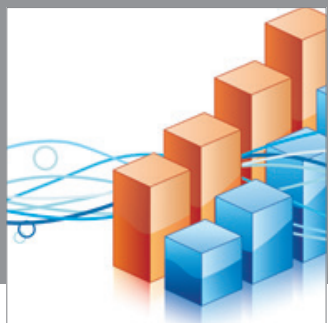

Advances in

Operations Research

mansans

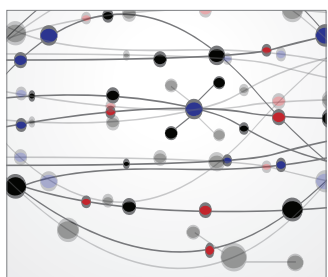

The Scientific World Journal
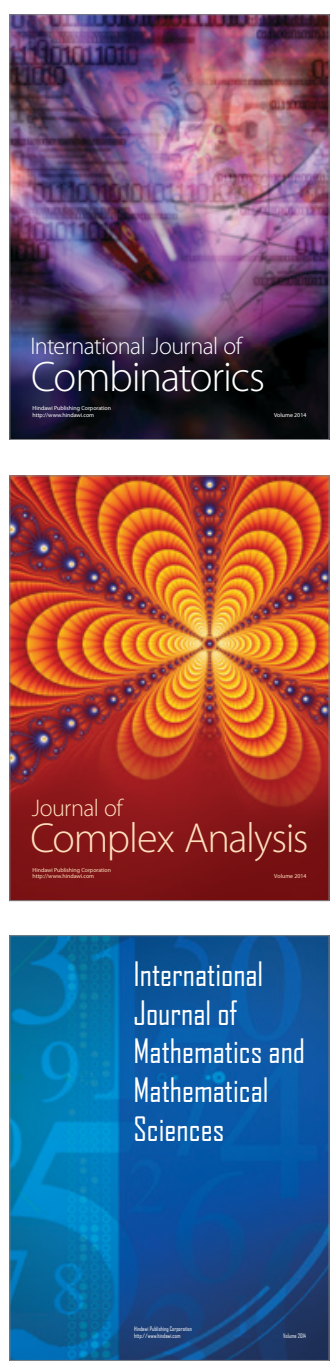
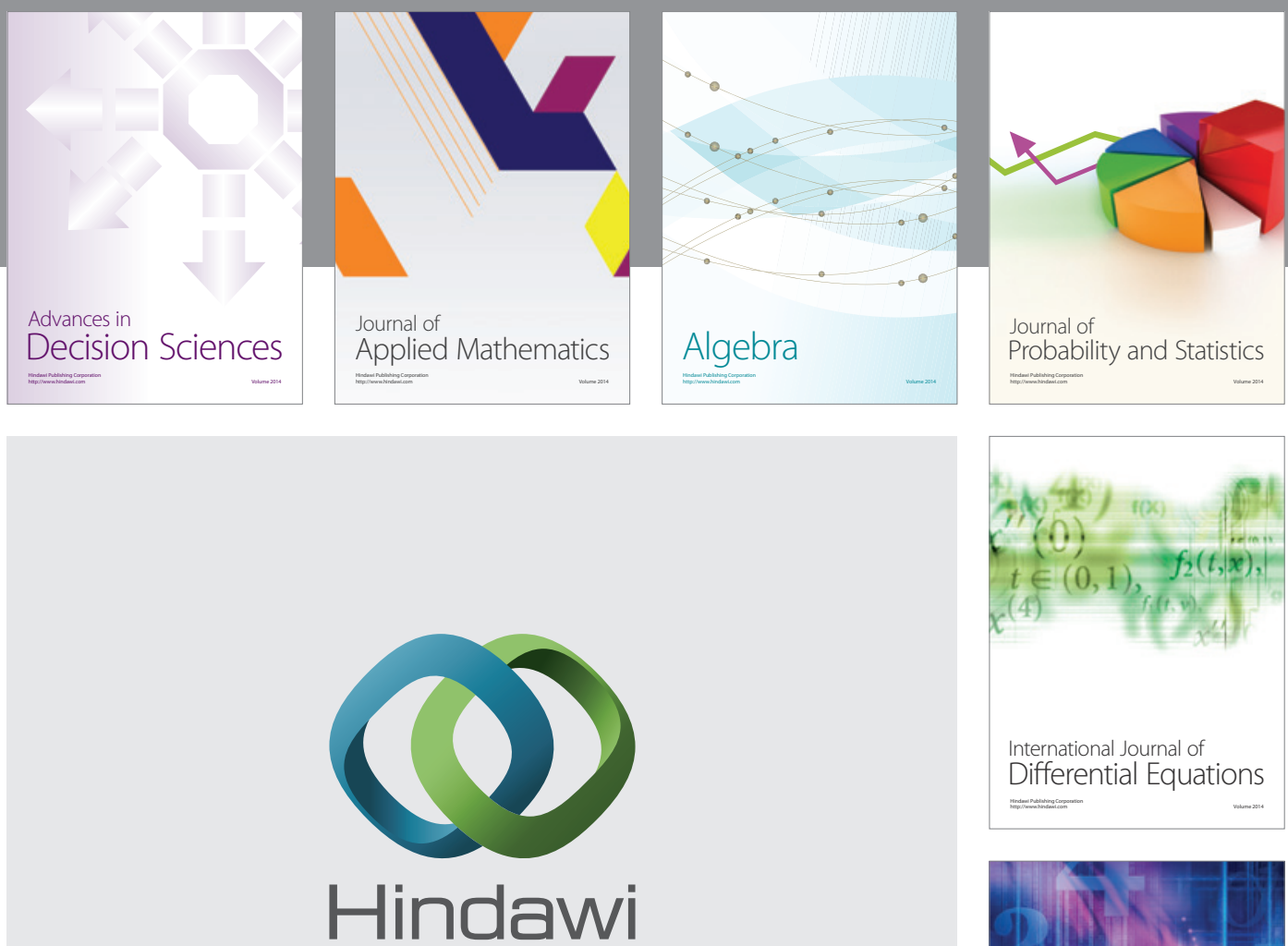

Submit your manuscripts at http://www.hindawi.com
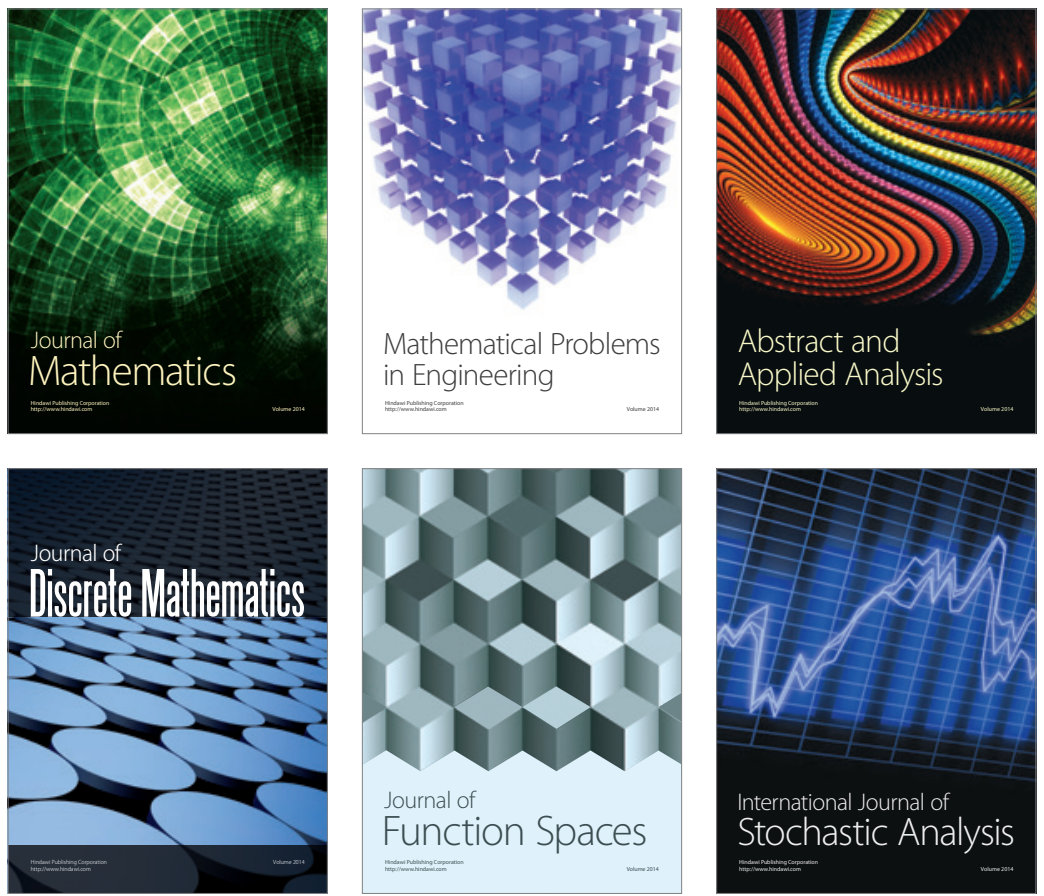

Journal of

Function Spaces

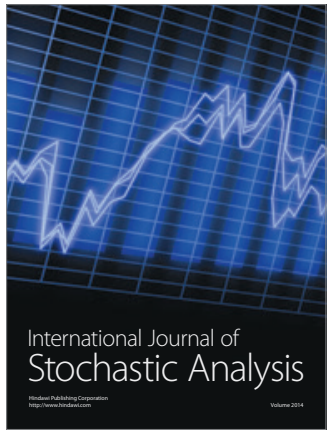

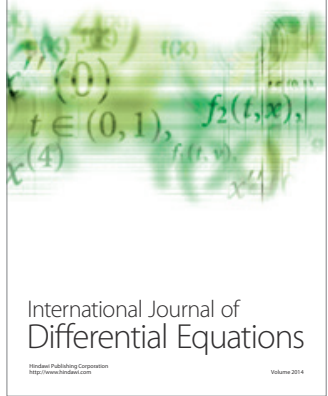
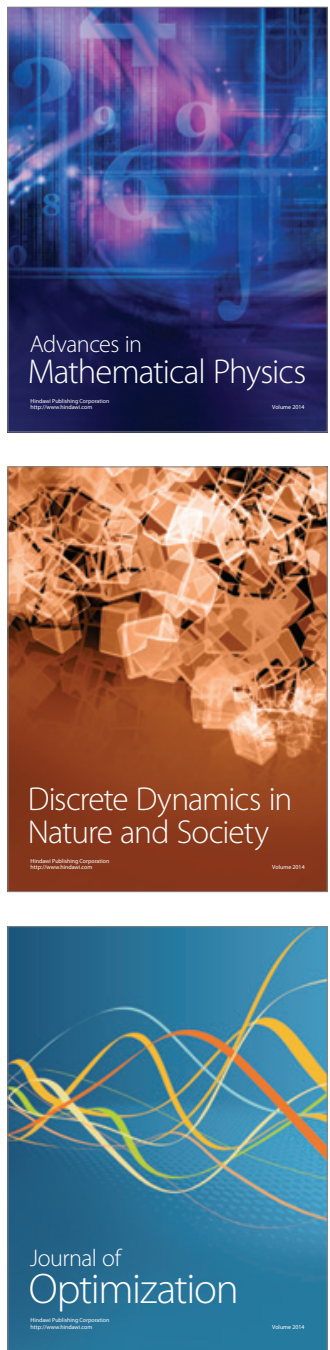